\title{
Aspectos sócio epidemiológicos e fatores associados a coinfecção Tuberculose/HIV- série temporal de 10 anos no Sudeste Brasileiro
}

\author{
Socio-epidemiological aspects and factors associated with tuberculosis / HIV co-infection - 10-year \\ time series in Southeast Brazil
}

Aspectos socioepidemiológicos y factores asociados a la coinfección tuberculosis / VIH - Serie

temporal de 10 años en el sureste de Brasil

Recebido: 17/02/2021 | Revisado: 23/02/2021 | Aceito: 25/02/2021 | Publicado: 06/03/2021

\author{
ORCID: https://orcid.org/0000-0001-5069-8812 \\ Universidade Estadual Paulista "Júlio de Mesquita Filho", Brasil \\ E-mail:clea.saliba-garbin@unesp.br \\ Julia Arruda Batista \\ ORCID: https://orcid.org/0000-0001-9621-7201 \\ Universidade Estadual Paulista "Júlio de Mesquita Filho", Brasil \\ E-mail: jarrudabaptista@gmail.com \\ Bruno Wakayama \\ ORCID: https://orcid.org/0000-0002-5152-3683 \\ Universidade Estadual Paulista "Júlio de Mesquita Filho",Brasil \\ E-mail:brunowakayama@gmail.com \\ Artênio José Saliba Garbin \\ ORCID: https://orcid.org/0000-0003-1566-681X \\ Centro Universitário Católico Salesiano Auxilium, Brasil \\ E-mail:tesjg@hotmail.com \\ Orlando Adas Saliba Júnior \\ ORCID: https://orcid.org/0000-0002-1175-7928 \\ Centro Universitário Católico Salesiano Auxilium, Brasil \\ E-mail:osalibajr@gmail.com \\ Artênio José Isper Garbin \\ ORCID: https://orcid.org/0000-0002-7017-8942 \\ Universidade Estadual Paulista "Júlio de Mesquita Filho", Brasil \\ E-mail:artenio.garbin@unesp.br
}

Clea Adas Saliba Garbin

\begin{abstract}
Resumo
Investigar o comportamento epidemiológico, aspectos sociodemográficos e fatores relacionados com o processo saúde-doença da coinfecção tuberculose/HIV nos 4 estados do Sudeste Brasileiro, em uma série temporal de 10 anos. Trata-se de um estudo descritivo retrospectivo com abordagem quantitativa dos casos confirmados/notificados de coinfecção no sudeste brasileiro. Para a coleta dos dados foi consultado o Sistema de Informação de Agravos no período de 2007 a 2017, utilizando o TabWin como ferramenta para análise das variáveis de interesse e as informações obtidas foram tabuladas com o auxílio do software EpiInfo 7.2 e Bioestat 5.3. Foram analisados 317.188 registros, sendo 45.552 casos de coinfecção tuberculose/HIV no sudeste brasileiro. Notou-se que $71.39 \%$ dos indivíduos com ambas afecções eram do sexo masculino, brancos (34.68\%) e faixa etária entre 30 a 39 anos(34.62\%) e que somente $1.78 \%$ haviam concluído o ensino superior.Quanto a manifestação clínica, a forma pulmonar foi mais frequente $(66.06 \%)$ e que grande parcela dos coinfectados se encontravam em situação de vulnerabilidade social em razão do uso de drogas ilícitas (14.49\%), alcoolismo(14.51\%) e tabagismo(6.95\%), resultando no abandono do tratamento (17.16\%) e óbitos por outras afecções(16.06\%).O comportamento epidemiológico da coinfecção tuberculose/HIV aumentou consideravelmente no período de 10 anos em virtude da falta de adesão a terapêutica da tuberculose, o que pode influenciar negativamente no resultado sorológico para o HIV, suscitando na elevada morbimortalidade em razão desses agravos em saúde.
\end{abstract}

Palavras-chave: Coinfecção; Infecções por HIV; Tuberculose.

\begin{abstract}
To investigate the epidemiological behavior, sociodemographic aspects and factors related to the health-disease process of tuberculosis / HIV coinfection in the 4 states of Southeast Brazil, in a 10-year time series. This is a retrospective descriptive study with a quantitative approach of confirmed / reported cases of coinfection in southeastern Brazil. For data collection, the Disease Information System was consulted from 2007 to 2017, using
\end{abstract}


TabWin as a tool for analysis of the variables of interest and the information obtained was tabulated with the help of EpiInfo 7.2 and Bioestat 5.3 softwares. A total of 317.188 records were analyzed, of which 45.552 cases of tuberculosis /HIV coinfection in southeastern Brazil. It was noted that $71.39 \%$ of individuals with both conditions (34.68\%) and aged between 30 to 39 years (34.62\%) and only $1.78 \%$ had completed higher education. Regarding the clinical manifestation, the pulmonary form was more frequent $(66.06 \%)$ and that a large proportion of the co-infected patients were socially vulnerable due to illicit drug use (14.49\%), alcoholism (14.51\%) and smoking (6.95\%), resulting in treatment abandonment $(17.16 \%)$ and deaths from other conditions (16.06\%). The epidemiological behavior of tuberculosis / HIV coinfection increased considerably over the 10-year period due to the lack of adherence to tuberculosis therapy, which can negatively influence the HIV serological result, leading to high morbidity and mortality due to these health problems.

Keywords: Coinfection; HIV Infections; Tuberculosis.

\begin{abstract}
Resumen
Investigar el comportamiento epidemiológico, los aspectos sociodemográficos y los factores relacionados con el proceso salud-enfermedad de la coinfección tuberculosis / VIH en los 4 estados del sureste brasileño, en una serie temporal de 10 años. Se trata de un estudio descriptivo retrospectivo con un enfoque cuantitativo de casos confirmados / notificados de coinfección en el sureste de Brasil. Para la recolección de datos se consultó el Sistema de Información de Enfermedades de 2007 a 2017, utilizando TabWin como herramienta de análisis de las variables de interés y la información obtenida se tabuló con la ayuda de los softwares EpiInfo 7.2 y Bioestat 5.3. Se analizaron un total de 317.188 registros, de los cuales 45.552 casos de coinfección tuberculosis /VIH en el sureste de Brasil. Se observó que el $71,39 \%$ de las personas con ambas afecciones eran hombres, blancos $(34,68 \%)$ y de 30 a 39 años $(34,62 \%)$ y solo el $1,78 \%$ había completado la educación superior. En cuanto a la manifestación clínica, la forma pulmonar fue más frecuente $(66,06 \%)$ y que una gran proporción de los pacientes coinfectados eran socialmente vulnerables por consumo de drogas ilícitas (14,49\%), alcoholismo (14,51\%) y tabaquismo $(6,95 \%)$, resultando en abandono del tratamiento $(17,16 \%)$ y muertes por otras condiciones $(16,06 \%)$. El comportamiento epidemiológico de la coinfección tuberculosis / VIH aumentó considerablemente en el período de 10 años debido a la falta de adherencia al tratamiento antituberculoso, lo que puede influir negativamente en el resultado serológico del VIH,generando una alta morbilidad y mortalidad por estos problemas de salud.
\end{abstract}

Palabras clave: Coinfeccíon; Infecciones por VIH; Tuberculosis.

\title{
1. Introdução
}

A tuberculose e o vírus da imunodeficiência humana (HIV/AIDS) são epidemias intimamente relacionadas e configuram mundialmente um problema de saúde pública. (Méndez, 2017; Martinson et al., 2011) A elevada prevalência dessas comorbidades implicam em desafios para os programas de controle da coinfecção tuberculose/HIV, dificultando à redução da incidência de ambas afecções. (Pawlowski et al., 2012; Bruchfeld et al., 2015; Brasil, 2019)

De acordo com recentes estudos, a coinfecção tuberculose/HIV exibe efeitos bidirecionais que coadjuvam uma combinação sinérgica de doenças. (Gesesew et al., 2015; Pawlowski et al., 2012) O HIV é considerado um fator de risco para a progressão da infecção latente para a tuberculose ativa, ao passo que, as infecções pelo vírus da tuberculose aumentam em vinte vezes a susceptibilidade ao HIV, e pode ocasionar a morte de indivíduos não tratados. (Kwan \& Ernst, 2011)

A transmissibilidade e disseminação destas afecções associam-se com diversos fatores propulsores, sendo o mais recorrente a resistência farmacológica aos medicamentos para a tuberculose e iniquidades em saúde. (Falana et al., 2018) Dessa forma, o diagnóstico/prevenção precoces são primordiais na definição das estratégias empregadas para minimizar os agravos e consecução de cura dos coinfectos. (Gopalan et al., 2016)

O conhecimento do status sorológico dos pacientes é crucial na determinação da manifestação clínica da tuberculose viabilizando o tratamento eficaz, tendo vista a cronicidade da infecção por HIV/AIDS. (Gopalan et al., 2016; Zhu et al., 2017) A terapia antirretroviral (TARV) é preconizada como recurso terapêutico em coinfectados, no entanto, segundo diretrizes da Organização Mundial de Saúde (OMS), o tratamento simultâneo das afecções podem suscitar em interações terapêuticas, comprometimento imunológico do paciente, além de falhos prognósticos. (Zhu et al., 2017)

O auge da epidemia tuberculose/HIV ocorreu nos anos 90, e desde então o vírus HIV tem sido apontado como a principal causa de insucesso no controle da tuberculose nas áreas endêmicas. (Cui et al., 2017). Em 2016, a Organização 
Mundial da Saúde (OMS) reportou que a epidemia de tuberculose foi a principal doença infecciosa com elevadas taxas de mortalidade (1,4 milhão de mortes) e com tendência crescente de indivíduos coinfectados (10\% ao ano). (Vachon et al., 2018; World Health Organization, 2017)

No Brasil, a incidência deste agravo é de aproximadamente 70 mil e ocasiona 4,5 mil mortes por ano. (Sousa et al., 2020) Considerando o arraigamento social das afecções, a OMS a fim de reduzir a incidência da coinfecção tuberculose/HIV redefiniu a classificação de países prioritários para o período de 2016 a 2020, no qual o Brasil ocupa a $20^{\circ}$ posição na classificação de carga da doença e $19^{\circ}$ quanto à coinfecção. (Sousa et al., 2020)

Tendo em vista o advento da epidemia HIV nos países endêmicos e o aumento significativo dos casos de tuberculose, o objetivo deste estudo foi investigar o comportamento epidemiológico, aspectos sociodemográficos e fatores relacionados com o processo saúde-doença da coinfecção tuberculose/HIV nos 4 estados do Sudeste Brasileiro, em uma série temporal de 10 anos.

\section{Metodologia}

Trata-se de um estudo descritivo retrospectivo com abordagem quantitativa dos casos confirmados/notificados de coinfecção tuberculose/HIV em 4 estados do Sudeste brasileiro.

Para a condução do estudo, foram consultados os registros do Sistema de Informação de Agravos (SINAN/DATASUS) no período de 2007 a 2017, utilizando o TabWin como ferramenta para análise das variáveis de interesse: características sociodemográficas (gênero, idade, escolaridade e raça) e perfil epidemiológico da coinfecção tuberculose/HIV (manifestação clínica, tratamento com a terapia antirretroviral (TARV), status sorológico e situação de encerramento das afecções). Dessa forma, os dados obtidos foram tabulados com auxílio do software Epi Info 7.2, e para análise descritiva das variáveis o software Bioestat 5.3 foi empregado.

O presente estudo foi aprovado pelo Comitê de Ética em Pesquisa, sendo realizada em conformidade aos preceitos éticos exigidos pela resolução 466/12 do Conselho Nacional de Saúde, consoante à Declaração de Helsinque.

\section{Resultados}

No período do estudo foram analisados 317.188 registros de casos confirmados/notificados de tuberculose no banco de dados do SINAN/DATASUS. Foi possível verificar 45.552 casos de coinfecção nos 4 estados do Sudeste, e que uma parcela pequena desses indivíduos fazia uso de Terapia antirretroviral- TARV (9.49\%). (Tabela 1)

De acordo com as características sociodemográficas, notou-se que grande parcela dos indivíduos com ambas afecções são do sexo masculino (71.39\%), com faixa etária compreendida entre 30 a 39 anos (34.62\%) e com predomínio da raça branca (34.68\%). (Tabela 1)

No que diz respeito a escolaridade, a maioria dos registros indicou que os indivíduos coinfectados não completaram o ensino fundamental $(24.61 \%)$ e que somente $1,78 \%$ concluíram o ensino superior. (Tabela 1 )

Na Tabela 1, percebeu-se que à manifestação clínica pulmonar da tuberculose foi mais frequente (66.06\%), seguido da extrapulmonar $(21.87 \%)$, enquanto que, $12.05 \%$ dos indivíduos coinfectados apresentavam ambas as formas de tuberculose. Quanto a situação de encerramento das infecções, $48.34 \%$ dos casos foram curados. Em contrapartida, $17.16 \%$ dos pacientes abandonaram o tratamento e $16.06 \%$ foram a óbito por outras doenças. (Tabela 1 ). 
Tabela 1. Características sociodemográficas em pacientes coinfectados pela Tuberculose/HIV nos estados do Sudeste Brasileiro, no período de 2007 a 2017.

\begin{tabular}{|c|c|c|c|c|c|c|c|c|c|c|c|c|c|c|c|c|c|c|c|c|}
\hline \multirow{3}{*}{$\begin{array}{c}\text { Característica } \\
\text { Sociodemográficas }\end{array}$} & \multicolumn{4}{|c|}{ SÃO PAULO } & \multicolumn{4}{|c|}{ RIO DE JANEIRO } & \multicolumn{4}{|c|}{ MINAS GERAIS } & \multicolumn{4}{|c|}{ ESPIRITO SANTO } & \multicolumn{4}{|c|}{ TOTAL } \\
\hline & \multicolumn{2}{|c|}{ Sem coinfecção } & \multicolumn{2}{|c|}{ Com coinfecção } & \multicolumn{2}{|c|}{ Sem coinfecção } & \multicolumn{2}{|c|}{ Com coinfecção } & \multicolumn{2}{|c|}{ Sem coinfecção } & \multicolumn{2}{|c|}{ Com coinfecção } & \multicolumn{2}{|c|}{ Sem coinfecção } & \multicolumn{2}{|c|}{$\begin{array}{c}\text { Com } \\
\text { coinfecção }\end{array}$} & Sem coin & cção & Com coi & nfecção \\
\hline & $\mathrm{n}$ & $f$ & $n$ & $f$ & $\mathrm{n}$ & $f$ & $\mathrm{n}$ & $f$ & $\mathrm{n}$ & $f$ & $\mathrm{n}$ & $f$ & $\mathrm{n}$ & $f$ & $\mathrm{n}$ & $f$ & $\mathrm{n}$ & $f$ & $\mathrm{n}$ & $f$ \\
\hline Sexo & & & & & & & & & & & & & & & & & & & & \\
\hline Feminino & 45.383 & 29,02 & 6.407 & 27,11 & 27.917 & 34,23 & 4.986 & 31,55 & 7.342 & 31,48 & 1.279 & 26,39 & 3.217 & 31,05 & 361 & 28,47 & 83.859 & 30,87 & 13.033 & 28,61 \\
\hline Masculino & 111.023 & 70,98 & 17.229 & 72,89 & 53.630 & 65,77 & 10.815 & 68,45 & 15.980 & 68,52 & 3.568 & 73,61 & 7.144 & 68,95 & 907 & 71,53 & 187.777 & 69,13 & 32.519 & 71,39 \\
\hline Idade & & & & & & & & & & & & & & & & & & & & \\
\hline$<4$ ano & 1089 & 0,7 & 39 & 0,17 & 751 & 0,92 & 107 & 0,68 & 150 & 0,64 & 14 & 0,29 & 109 & 1,05 & 11 & 0,87 & 2099 & 0,77 & 171 & 0,38 \\
\hline 5 a 9 & 793 & 0,51 & 47 & 0,2 & 382 & 0,47 & 49 & 0,31 & 87 & 0,37 & 13 & 0,27 & 40 & 0,39 & 1 & 0,08 & 1302 & 0,48 & 110 & 0,24 \\
\hline 10 a 19 & 11.102 & 7,1 & 469 & 1,99 & 6716 & 8,24 & 485 & 3,07 & 1.190 & 5,10 & 76 & 1,57 & 809 & 7,81 & 31 & 2,44 & 19817 & 7,30 & 1061 & 2,33 \\
\hline 20 a 29 & 44.280 & 28,31 & 4.084 & 17,28 & 20.299 & 24,89 & 3.329 & 21,07 & 4.134 & 17,73 & 757 & 15,62 & 2.748 & 26,52 & 262 & 20,66 & 71461 & 26,31 & 8432 & 18,51 \\
\hline 30 a 39 & 34.816 & 22,26 & 8.421 & 35,63 & 16.192 & 19,86 & 5.183 & 32,80 & 5.007 & 21,47 & 1.760 & 36,31 & 2.166 & 20,91 & 405 & 31,94 & 58181 & 21,42 & 15769 & 34,62 \\
\hline 40 a 49 & 26.769 & 17,12 & 7.064 & 29,89 & 14.544 & 17,84 & 4.098 & 25,94 & 4.945 & 21,20 & 1.375 & 28,37 & 1.876 & 18,11 & 330 & 26,03 & 48134 & 17,72 & 12867 & 28,25 \\
\hline 50 a 59 & 20.862 & 13,34 & 2.699 & 11,42 & 12.934 & 15,86 & 1.933 & 12,23 & 4.039 & 17,32 & 624 & 12,87 & 1.473 & 14,22 & 178 & 14,04 & 39308 & 14,47 & 5434 & 11,93 \\
\hline 60 a 69 & 10.338 & 6,61 & 607 & 2,57 & 6.380 & 7,82 & 505 & 3,20 & 2.197 & 9,42 & 170 & 3,51 & 722 & 6,97 & 41 & 3,23 & 19637 & 7,23 & 1323 & 2,90 \\
\hline 70 a 79 & 4.438 & 2,84 & 132 & 0,56 & 2.502 & 3,07 & 89 & 0,56 & 1.133 & 4,86 & 50 & 1,03 & 303 & 2,92 & 9 & 0,71 & 8376 & 3,08 & 280 & 0,61 \\
\hline $80+$ & 1.431 & 0,91 & 22 & 0,09 & 811 & 0,99 & 17 & 0,11 & 440 & 1,89 & 8 & 0,17 & 115 & 1,11 & 0 & 0,00 & 2797 & 1,03 & 47 & 0,10 \\
\hline Sem informação & 488 & 0,31 & 52 & 0,22 & 36 & 0,04 & 6 & 0,04 & & & & & & & 0 & & 524 & 0,19 & 58 & 0,13 \\
\hline Escolaridade & & & & & & & & & & & & & & & & & & & & \\
\hline Analfabeto & 1.872 & 1,2 & 157 & 0,66 & 1.027 & 1,26 & 212 & 1,34 & 493 & 2,11 & 60 & 1,24 & 319 & 3,08 & 37 & 2,92 & 3.711 & 1,37 & 466 & 1,02 \\
\hline Fund. Inc & 42.985 & 27,48 & 6.365 & 26,93 & 17536 & 21,50 & 3710 & 23,48 & 4886 & 20,95 & 822 & 16,96 & 2795 & 26,98 & 313 & 24,68 & 68.202 & 25,11 & 11210 & 24,61 \\
\hline $\begin{array}{l}\text { Fund. Com - Méd. } \\
\text { Inc }\end{array}$ & 33.874 & 21,66 & 4.813 & 20,36 & 6843 & 8,39 & 1417 & 8,97 & 1370 & 5,87 & 309 & 6,38 & 991 & 9,56 & 85 & 6,70 & 43.078 & 15,86 & 6624 & 14,54 \\
\hline $\begin{array}{l}\text { Med. Com - Sup. } \\
\text { Inc }\end{array}$ & 8.590 & 5,49 & 1.131 & 4,79 & 6157 & 7,55 & 1145 & 7,25 & 1223 & 5,24 & 162 & 3,34 & 799 & 7,71 & 78 & 6,15 & 16.769 & 6,17 & 2516 & 5,52 \\
\hline Sup. Comp & 3.277 & 2,1 & 447 & 1,91 & 1.581 & 1,94 & 289 & 1,83 & 337 & 1,44 & 59 & 1,22 & 170 & 1,64 & 16 & 1,26 & 5.365 & 1,98 & 811 & 1,78 \\
\hline Sem inf. & 65.808 & 42,08 & 10.723 & 45,35 & 48.403 & 59,36 & 9028 & 57,14 & 15013 & 64,37 & 3435 & 70,87 & 5.287 & 51,03 & 739 & 58,28 & 134.511 & 49,52 & 23925 & 52,52 \\
\hline Raça-cor da pele & & & & & & & & & & & & & & & & & & & & \\
\hline Branca & 63.103 & 40,35 & 9.522 & 40,29 & 27.568 & 33,81 & 4.587 & 29,03 & 7.497 & 32,15 & 1.417 & 29,23 & 2.738 & 26,43 & 272 & 21,45 & 100.906 & 37,15 & 15.798 & 34,68 \\
\hline Preta & 14.849 & 9,49 & 2.577 & 10,9 & 16.245 & 19,92 & 3.784 & 23,95 & 4.287 & 18,38 & 936 & 19,31 & 1.715 & 16,55 & 227 & 17,90 & 37.096 & 13,66 & 7.524 & 16,52 \\
\hline Amarela & 1.254 & 0,8 & 75 & 0,32 & 591 & 0,72 & 100 & 0,63 & 193 & 0,83 & 28 & 0,58 & 45 & 0,43 & 7 & 0,55 & 2.083 & 0,77 & 210 & 0,46 \\
\hline Parda & 46.510 & 29,74 & 6.341 & 26,83 & 29.741 & 36,47 & 5.578 & 35,30 & 8.884 & 38,09 & 1.684 & 34,74 & 5.243 & 50,60 & 651 & 51,34 & 90.378 & 33,27 & 14.254 & 31,29 \\
\hline Indigena & 808 & 0,52 & 32 & 0,14 & 160 & 0,20 & 31 & 0,20 & 52 & 0,22 & 11 & 0,23 & 18 & 0,17 & 1 & 0,08 & 1.038 & 0,38 & 75 & 0,16 \\
\hline Sem inf. & 29.882 & 19,11 & 5.089 & 21,53 & 7.242 & 8,88 & 1.721 & 10,89 & 2.409 & 10,33 & 771 & 15,91 & 602 & 5,81 & 110 & 8,68 & 40.135 & 14,78 & 7.691 & 16,88 \\
\hline TARV & & & & & & & & & & & & & & & & & & & & \\
\hline Sim & - & - & 1.452 & 6,15 & - & - & 2.051 & 12,98 & - & - & 639 & 13,18 & - & - & 183 & 14,43 & - & - & 4.325 & 9,49 \\
\hline Não & - & - & 470 & 1,99 & - & - & 935 & 5,92 & - & - & 284 & 5,86 & - & - & 89 & 7,02 & - & - & 1.778 & 3,90 \\
\hline Sem inf. & - & - & 21.714 & 91,87 & - & - & 12.815 & 81,10 & - & - & 3.924 & 80,96 & - & - & 996 & 78,55 & - & - & 39.449 & 86,60 \\
\hline Tipo/Manifestação & & & & & & & & & & & & & & & & & & & & \\
\hline Pulmonar & 131.372 & 83,98 & 15.084 & 63,83 & 70.580 & 86,55 & 11.397 & 72,13 & 18.814 & 80,67 & 2.816 & 58,10 & 8.695 & 83,92 & 795 & 62,70 & 229.461 & 84,47 & 30.092 & 66,06 \\
\hline Extra-pulmonar & 21.008 & 13,43 & 5.613 & 23,75 & 8.686 & 10,65 & 2.730 & 17,28 & 3.742 & 16,04 & 1.318 & 27,19 & 1.324 & 12,78 & 302 & 23,82 & 34.760 & 12,80 & 9.963 & 21,87 \\
\hline Ambos & 4.022 & 2,57 & 2.934 & 12,41 & 2.281 & 2,80 & 1.673 & 10,59 & 766 & 3,28 & 713 & 14,71 & 342 & 3,30 & 171 & 13,49 & 7.411 & 2,73 & 5.491 & 12,05 \\
\hline Sem inf. & 4 & 0,01 & 5 & 0,02 & 0 & 0,00 & 1 & 0,01 & & & & & 0 & & 0 & & 4 & 0,00 & 6 & 0,01 \\
\hline Situação dos casos & & & & & & & & & & & & & & & & & & & & \\
\hline Cura & 126.728 & 81,03 & 12.915 & 54,64 & 55.766 & 68,39 & 6.365 & 40,28 & 17.296 & 74,16 & 2.110 & 43,53 & 8.309 & 80,19 & 628 & 49,53 & 208.099 & 76,61 & 22.018 & 48,34 \\
\hline Abandono & 14.170 & 9,06 & 4.276 & 18,09 & 9.701 & 11,90 & 2.377 & 15,04 & 2.560 & 10,98 & 1006 & 20,76 & 792 & 7,64 & 160 & 12,62 & 27.223 & 10,02 & 7.819 & 17,16 \\
\hline $\begin{array}{l}\text { Óbito por } \\
\text { Tuberculose }\end{array}$ & 3.433 & 2,19 & 226 & 0,96 & 2.157 & 2,65 & 1.858 & 11,76 & 728 & 3,12 & 255 & 5,26 & 250 & 2,41 & 99 & 7,81 & 6.568 & 2,42 & 2.438 & 5,35 \\
\hline $\begin{array}{l}\text { Óbito (out. } \\
\text { doenças) }\end{array}$ & 3.153 & 2,02 & 4.870 & 20,6 & 1.341 & 1,64 & 1.141 & 7,22 & 759 & 3,25 & 1.066 & 21,99 & 167 & 1,61 & 238 & 18,77 & 5.420 & 2,00 & 7.315 & 16,06 \\
\hline Transferência & 1.153 & 0,74 & 203 & 0,86 & 5.246 & 6,43 & 1.942 & 12,29 & 739 & 3,17 & 199 & 4,11 & 468 & 4,52 & 102 & 8,04 & 7.606 & 2,80 & 2.446 & 5,37 \\
\hline Sem inf. & 7.769 & 4,97 & 1.146 & 4,85 & 7336 & 9,00 & 2118 & 13,40 & 1240 & 5,32 & 211 & 4,35 & 375 & 3,62 & 41 & 3,23 & 16.720 & 6,16 & 3.516 & 7,72 \\
\hline Total & 156.406 & 100 & 23.636 & 100 & 81.547 & 100 & 15.801 & 100 & 23.322 & 100 & 4.847 & 100 & 10.361 & 100 & 1.268 & 100 & 271.636 & 100 & 45.552 & 100 \\
\hline
\end{tabular}

Fonte: SINAN/DATASUS

Notou-se no presente estudo que no estado de São Paulo, 11002 indivíduos coinfectados pela tuberculose/HIV se encontravam em situação de vulnerabilidade social. O uso de drogas ilícitas (14.49\%), alcoolismo (14.51\%) e o tabagismo (6.95\%) foram circunstâncias mais recorrente quando comparadas com os outros estados do sudeste brasileiro. (Tabela 2) 
Tabela 2. Situação de vulnerabilidade dos indivíduos coinfectados nos estados do sudeste brasileiro.

\begin{tabular}{l|c|c|c|c|c}
\hline \begin{tabular}{c} 
Situação de \\
Vulnerabilida \\
\multicolumn{1}{c|}{ de }
\end{tabular} & SÃO PAULO & $\begin{array}{c}\text { RIO DE } \\
\text { JANEIRO }\end{array}$ & $\begin{array}{c}\text { MINAS } \\
\text { GERAIS }\end{array}$ & $\begin{array}{c}\text { ESPIRITO } \\
\text { SANTO }\end{array}$ & TOTAL \\
\cline { 2 - 6 } & $\mathbf{n}$ & $\mathbf{n}$ & $\mathbf{n}$ & $\mathbf{n}$ & $\mathbf{n}$ \\
\hline Drogas ilícitas & 3426 & 914 & 397 & 92 & 4829 \\
Alcoolismo & 3430 & 2192 & 1315 & 300 & 7237 \\
Tabagismo & 1643 & 846 & 372 & 106 & 2967 \\
Morador de & 1245 & 282 & 133 & 29 & 1689 \\
rua & 1005 & 346 & 229 & 55 & 1635 \\
Presidiário & 170 & 36 & 5 & 6 & 217 \\
Profíssional & 75 & 111 & 13 & 3 & 202 \\
Gestante & 8 & 16 & 3 & 0 & 27 \\
Hosp. & 11002 & 4743 & 2467 & 591 & 18803 \\
\hline Psiquiátrico & & & & & \\
\hline
\end{tabular}

Fonte: SINAN/DATASUS

Em 2017, o número de indivíduos infectados por tuberculose foi de 21.876. Diante da confirmação/notificação desses casos, $88.90 \%$ realizaram o teste para diagnóstico do HIV, dentre os quais, $10.35 \%$ se apresentaram coinfectados pelas epidemias de tuberculose/HIV. (Tabela 3) Por meio dos dados apresentados no Gráfico 1, evidenciou-se que os casos de Tuberculose no Brasil no mesmo ano foi de 90.682, sendo 41.400 somente na região Sudeste do país. 
Tabela 3. Caos notificados de tuberculose, realização de teste antiHIV e status sorológico no estado de São Paulo, Brasil.

\begin{tabular}{|c|c|c|c|c|c|c|c|c|c|c|}
\hline \multicolumn{3}{|c|}{ TUBERCULOSE } & \multicolumn{4}{|c|}{ TESTE HIV } & \multicolumn{4}{|c|}{ SOROLOGIA } \\
\hline & & & \multicolumn{2}{|c|}{ Não realizado } & \multicolumn{2}{|c|}{ Realizado } & \multicolumn{2}{|c|}{ Negativo } & \multicolumn{2}{|c|}{ Positivo } \\
\hline $\begin{array}{c}\text { Ano } \\
\text { diagnóstico }\end{array}$ & $\mathbf{n}$ & $\mathbf{f}$ & $\mathbf{n}$ & $\mathbf{f}$ & $\mathbf{n}$ & $\mathbf{f}$ & $\mathbf{n}$ & $\mathbf{f}$ & $\mathbf{n}$ & f \\
\hline 2007 & 17584 & 8,13 & 4099 & 23,31 & 13485 & 76,69 & 11137 & 82,59 & 2348 & 17,41 \\
\hline 2008 & 18440 & 8,72 & 3959 & 21,47 & 14481 & 78,53 & 12202 & 84,26 & 2279 & 15,74 \\
\hline 2009 & 17855 & 8,44 & 3459 & 19,37 & 14396 & 80,63 & 12223 & 84,91 & 2173 & 15,09 \\
\hline 2010 & 18245 & 8,63 & 3092 & 16,95 & 15153 & 83,05 & 12867 & 84,91 & 2286 & 15,09 \\
\hline 2011 & 19011 & 8,99 & 3106 & 16,34 & 15905 & 83,66 & 13650 & 85,83 & 2255 & 14,18 \\
\hline 2012 & 18577 & 8,78 & 2958 & 15,92 & 15619 & 84,08 & 13526 & 86,6 & 2093 & 13,4 \\
\hline 2013 & 19722 & 9,32 & 2387 & 12,1 & 17335 & 87,9 & 15161 & 87,46 & 2174 & 12,54 \\
\hline 2014 & 19576 & 9,25 & 2065 & 10,55 & 17511 & 89,45 & 15405 & 87,97 & 2106 & 12,03 \\
\hline 2015 & 20377 & 9,63 & 1994 & 9,79 & 18383 & 90,21 & 16378 & 89,09 & 2005 & 10,91 \\
\hline 2016 & 20265 & 9,58 & 1938 & 9,56 & 18327 & 90,44 & 16423 & 89,61 & 1904 & 10,39 \\
\hline 2017 & 21876 & 10,34 & 2429 & 11,1 & 19447 & 88,9 & 17434 & 89,65 & 2013 & 10,35 \\
\hline Total & 211528 & 100 & 31486 & 14,89 & 180042 & 85,11 & 156406 & 86,87 & 23636 & 13,12 \\
\hline
\end{tabular}

Fonte: SINAN/DATASUS.

Da mesma forma, nesta investigação tornou-se possível analisar que há elevadas taxas de coinfecção tuberculose/HIV na região Sudeste (3.914) quando comparado com a média do Brasil (1.969). (Gráfico 1). 
Gráfico 1. Comparação das médias de tuberculose e coinfecção TUBERCULOSE/HIV no Brasil e na Região Sudeste no período de 2007 a 2017.

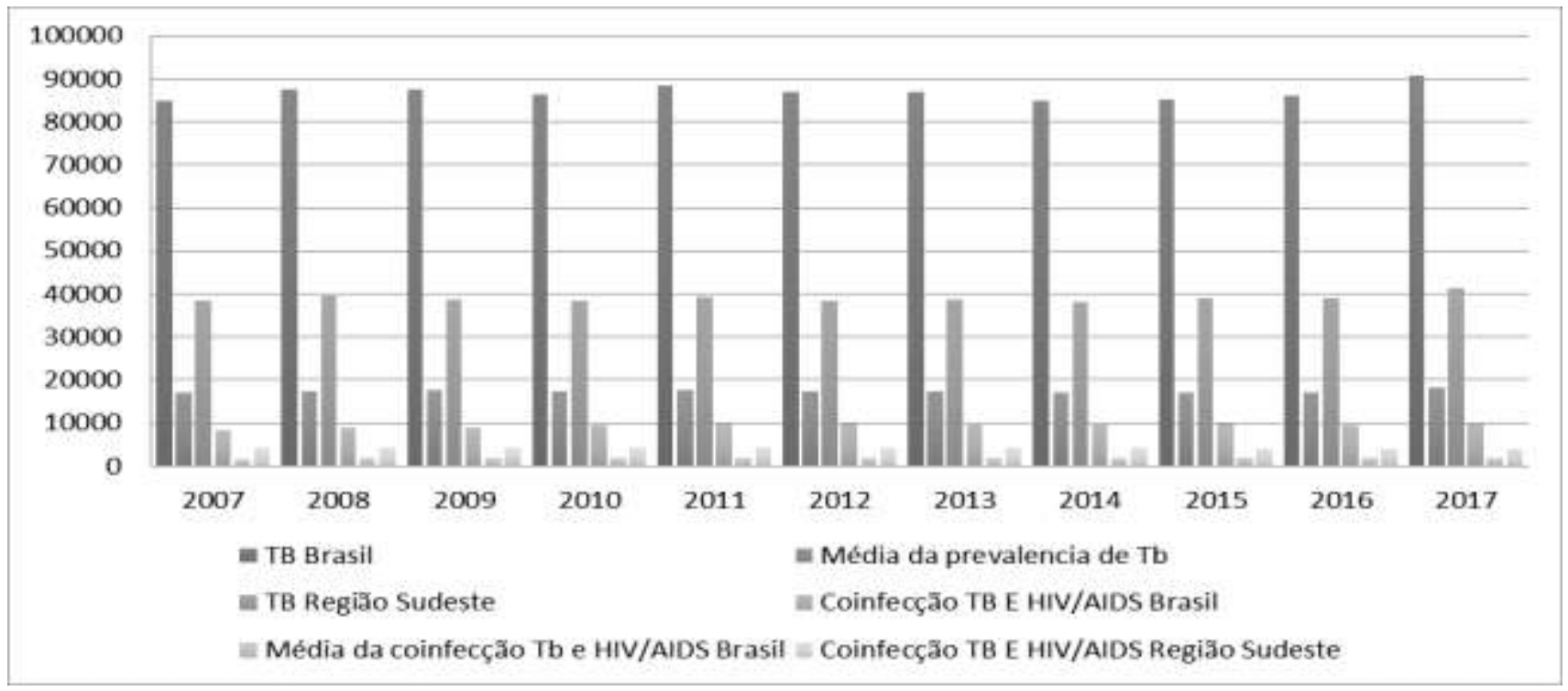

Fonte: SINAN/DATASUS.

\section{Discussões}

No decorrer dos anos as epidemias de tuberculose e HIV/AIDS vêm alcançando números crescentes e em razão da diversidade epidemiológica analisada em diferentes estados do Sudeste Brasileiro, novos estudos sobre a coinfecção tuberculose/HIV com abordagem nas necessidades regionais são pertinentes para a elaboração das políticas públicas de saúde. (Amorim \& Schlemper, 2019)

No presente estudo, na análise temporal de 10 anos, verificou-se que dos 45.552 casos confirmados/notificados, 71.39\% dos pacientes coinfectados eram do sexo masculino e com faixa etária compreendida entre 30 a 39 anos (34.62\%). O fato de haver predomínio de indivíduos masculino coinfectados, pode estar associado com o adoecimento de mais homens pela tuberculose, tendo em vista que a exposição ao bacilo, e a trajetória da doença são dissemelhante entre os gêneros. (Amorim \& Schlemper, 2019; Cardoso et al., 2018; Liberato et al.,2018). Além disso, notou-se que a baixa frequência de casos de coinfecção tuberculose/HIV em indivíduos com idade inferior a 19 anos (2.95\%), pode ser atribuída a dificuldade na confirmação bacteriológica em razão da tuberculose na infância não apresentar sinais/sintomas específicos. (Amorim \& Schlemper, 2019)

De acordo com estudos, o nível de escolaridade influencia na compreensão individual das afecções, tornando os coinfectados mais relutantes a aderirem aos tratamentos, favorecendo a transmissibilidade viral das doenças. (Amorim \& Schlemper, 2019; Liberato et al.,2018) Ademais, a baixa escolaridade retrata o cenário brasileiro caracterizado pela predominância de analfabetos funcionais, assim como nesta investigação, que uma parcela considerável dos pacientes não concluiu o ensino fundamental (24.61\%). (Vendramini et al., 2010)

No que diz respeito ao tipo/manifestação clínica da tuberculose, por meio dos dados obtidos no DATASUS/SINAM pode-se verificar que a doença foi mais recorrente em sua forma pulmonar em todos os estados do Sudeste Brasileiro, totalizando $66.06 \%$ dos casos, enquanto que $21.87 \%$ apresentaram a forma extrapulmonar e $12.05 \%$ uma combinação de pulmonar/extrapulmonar. Em consonância com outro estudo, a predominância da tuberculose pulmonar pode ser justificada em razão da instalação do Mycobacterium tuberculosis, bactéria estritamente aeróbica, preferencialmente em áreas com elevadas concentraç̃oes de oxigênio como o pulmão. (Amorim \& Schlemper, 2019) 
Considerando a relevância da terapia antirretroviral no tratamento da imunodeficiência humana, estudos recentes relataram que a TARV deve ser iniciada em até 8 semanas após a terapêutica anti-tuberculose para que as afecções não sejam exacerbadas e os resultados sejam considerados satisfatórios. (Gesesew et al., 2016; Bruchfeld et al., 2015) Gopalan et al. reforçou em seu estudo que apenas um terço da população coinfectada fazia uso da TARV e ressaltou que este recurso quando iniciado simultaneamente em pacientes coinfectados pode induzir interações medicamentosas, o aparecimento de síndromes inflamatórias imune e um aumento de riscos tóxicos. (Gopalan et al., 2016; Zhu et al., 2017) A falta de adesão a TARV pode ser explicada em razão do estigma da doença pelo fato da terapêutica ser disponibilizada em centros especialidades de controle/prevenção destes agravos, impactando não somente no resultado clínico, mas também nas crenças sobre o tratamento e no estado de saúde geral. (Gopalan et al., 2016; Frasca et al., 2014) Na presente pesquisa, somente 9.49\% dos pacientes coinfectados realizavam a TARV, e a grande maioria se concentrava nos estados do Rio de Janeiro (12.98\%) e São Paulo $(6.15 \%)$.

No que diz respeito às doenças que emergem das iniquidades sociais, o status de saúde dos indivíduos coinfectados pela tuberculose/HIV é imprescindível para compreensão das necessidade e cuidados em saúde. Neste contexto, a vulnerabilidade social oportuniza o adoecimento/óbito desta população, além de resultar na marginalização quanto a dificuldade de acesso aos serviços de saúde, dificultando assim, o tratamento das afecções. (Brunello et al., 2011; Oliveira et al., 2020)

Verificou-se neste estudo que as situações de vulnerabilidade mais frequentes foram o uso de drogas ilícitas (14.49\%), alcoolismo $(14.51 \%)$ e o tabagismo (6.95\%). Estudos atuais sugerem que entre os usuários de drogas ilícitas a infecção e a progressão para tuberculose ativa associam-se a diversos fatores que podem facilitar a transmissibilidade e a disseminação da doença, como o estilo de vida, o isolamento em ambientes para uso de drogas e o compartilhamento de cachimbos. (Brunello et al., 2011; Silva, 2018) Em relação ao consumo de álcool, as complicações do uso como danos no fígado e deficiências nutricionais aumentam a susceptibilidade a tuberculose em razão da depressão do sistema imunológico. (Silva, 2018). No entanto, a situação de vulnerabilidade mais impactante para a tuberculose é o tabagismo, que por sua vez eleva a taxa de mortalidade em 9 vezes em pacientes infectados tendo em vista que a fumaça do cigarro promove disfunção ciliar e a imunossupressão favorecendo a instalação do Mycobacterium tuberculosis. (Silva, 2018)

Em se tratando de doenças que afetam o sistema imunológico, a coinfecção pela tuberculose e o vírus do HIV/AIDS não produzem somente uma combinação de sinais/sintomas, mas se intensificam entre si. (Liberato et al., 2004) No presente estudo, percebeu-se que a situação de cura dos pacientes que apresentavam apenas um dos agravos foi maior nos 4 estados do sudeste brasileiro. Em contrapartida, em pacientes coinfectados o encerramento da doença em razão da cura foi consideravelmente menor em comparação com indivíduos que apresentavam somente a tuberculose. Esses resultados corroboram com estudo de Liberato et.al sendo suportado pela premissa de mudança no padrão clínico usual da tuberculose em pacientes HIV positivo, tornando o diagnóstico dificultado para esta afecção. (Liberato et al., 2004)

Quanto as demais situações de desfecho, houve uma taxa elevada de abandono do tratamento (17.16\%), seguidos por óbitos por outra doença $(16.06 \%)$ e óbitos por tuberculose $(5.35 \%)$. Estudos ressaltam que o tratamento em indivíduos imunossuprimidos difere daquele em imunocompetentes, explicando assim, a resistência terapêutica anti tuberculose, resultando na taxa de mortalidade aumentada em duas vezes em coinfectados. Além disso, o uso simultâneo de muitos fármacos e os efeitos colaterais fundamentam a razão de maior abandono do tratamento. (Cardoso et al., 2018; Liberato et al., 2004; Silva et al., 2020)

Quando comparado com a média de coinfecção tuberculose/HIV no Brasil, o Sudeste Brasileiro apresentou números elevados de casos (3.914), explicada pela heterogeneidade na distribuição das epidemias ocasionadas pelas desigualdades intra-regionais. (Vendramini et al., 2010) Neste sentido, faz-se necessário o planejamento de novas políticas públicas a fim de 
orientar os pacientes coinfectados sobre ambas afecções, minimizando a diversidade epidemiológica determinantes no processo saúde-doença.

\section{Conclusão}

Conclui-se que o comportamento epidemiológico da coinfecção tuberculose/HIV aumentou consideravelmente no período de 10 anos. Os indivíduos coinfectados, em sua maioria eram homens brancos, com idade entre 30 a 39 anos e que não concluíram o ensino fundamental. Já em relação aos fatores relacionados às doenças, a tuberculose pulmonar foi a manifestação clínica mais recorrente, e grande parcela da população dos 4 estados não faziam a terapêutica com a TARV, o que pode influenciar negativamente no resultado sorológico para o HIV, resultando em números elevados de morbimortalidades pela própria tuberculose ou outros agravos.

\section{Agradecimentos}

O presente trabalho foi realizado com apoio da Coordenação de Aperfeiçoamento de Pessoal de Nível Superior- Brasil (CAPES) - Código de Financiamento 001.

\section{Referências}

Amorim, L. T., \& Schlemper Junior, B. R. (2019). HIV/AIDS in small cities in Midwest Santa Catarina, south of Brazil: Clinical and epidemiological aspects, opportunistic infections. Revista da Sociedade Brasileira de Medicina Tropical, 52, e20180430.

Bruchfeld, J., Correia-Neves, M., \& Källenius, G. (2015). Tuberculosis and HIV coinfection. Cold Spring Harbor perspectives in medicine, 5(7), a017871.

Brunello, M. E. F., Chiaravalotti, N. F., Arcência, R. A., Andrade, R. L. P., Magnabosco, G. T., \& Villa, T. C (2011) Áreas de vulnerabilidade para coinfecção HIV-aids/TUBERCULOSE em Ribeirão Preto, SP. Revista de Saúde Pública, 45, 556-563.

Cardoso, L. C., Melquiades, R., Magalhães, J. A., Carvalho, S. S., da Rosa, G., Merlini, L. S., \& Julião, V. A. P. (2018). Aspectos epidemiológicos dos pacientes notificados com tuberculose na microrregião de Umuarama-noroeste paranaense de 2009 a 2014. Arquivos de Ciências da Saúde da UNIPAR, 22(3), $157-163$.

Cui, Z., Lin, M., Nie, S., \& Lan, R. (2017). Risk factors associated with Tuberculosis (TB) among people living with HIV/AIDS: A pair-matched case-control study in Guangxi, China. PLoS One, 12(3), e0173976.

Falana, A., Akpojiyovwi, V., Sey, E., Akpaffiong, A., Agumbah, O., Chienye, S., \& Salihu, H. M. (2018). Hospital length of stay and cost burden of HIV, tuberculosis, and HIV-tuberculosis coinfection among pregnant women in the United States. American journal of infection control, 46(5), 564-570.

Frasca, K., \& Cohn, J. (2014). Integration of HIV and tuberculosis in the community. Journal of the International Association of Providers of AIDS Care (JIAPAC), 13(6), 534-538.

Gesesew, H., Tsehaineh, B., Massa, D., Tesfay, A., Kahsay, H., \& Mwanri, L. (2016). The prevalence and associated factors for delayed presentation for HIV care among tuberculosis/HIV co-infected patients in Southwest Ethiopia: a retrospective observational cohort. Infectious diseases of poverty, 5(1), 1-10.

Gopalan, N., Chandrasekaran, P., Swaminathan, S., \& Tripathy, S. (2016). Current trends and intricacies in the management of HIV-associated pulmonary tuberculosis. AIDS research and therapy, 13(1), 1-19.

Kwan, C. K., \& Ernst, J. D. (2011). HIV and tuberculosis: a deadly human syndemic. Clinical microbiology reviews, 24(2), $351-376$.

Liberato, I. R. D. O., Albuquerque, M. D. F. P., Campelo, A. R. L., \& Melo, H. R. L. D. (2004). Características da tuberculose pulmonar em pacientes com sorologia positiva e negativa para o HIV em uma região do Nordeste do Brasil. Revista da Sociedade Brasileira de Medicina Tropical, 37(1), 46-50.

Martinson, N. A., Hoffmann, C. J., \& Chaisson, R. E. (2011). Epidemiology of tuberculosis and HIV: recent advances in understanding and responses. Proceedings of the American Thoracic Society, 8(3), 288-293.

Méndez-Samperio, P. (2017). Diagnosis of tuberculosis in HIV co-infected individuals: current status, challenges and opportunities for the future. Scandinavian journal of immunology, 86(2), 76-82.

Ministério da Saúde. Manual de Recomendações para o controle da Tuberculose no Brasil. Secretária de Vigilância em Saúde.

Oliveira, A. V. S. de, Alfonso, A. D., Miranda, R. S. de, \& oliveira, T. R. S. de. (2020). Epidemiological profile of tuberculosis in the northeast of Brazil: temporary series from 2008 to 2018. Research, Society and Development, 9(2), e108922129.

Pawlowski, A., Jansson, M., Sköld, M., Rottenberg, M .E., \& Källeniu, G. (2012) Tuberculosis and HIV co-infection. PLoS Pathogens, 8, e1002464. 
Research, Society and Development, v. 10, n. 3, e6910313062, 2021

(CC BY 4.0) | ISSN 2525-3409 | DOI: http://dx.doi.org/10.33448/rsd-v10i3.13062

Silva, D. R. (2018). Fatores de risco para tuberculose: diabetes, tabagismo, álcool e uso de outras drogas. Jornal Brasileiro de Pneumologia, 44, 145-152.

Silva, L. F. da, Carvalho, N. S. de, Souza, B. W. L. de, Castro, R. da C., Feitosa, A. P. A. R., \& Martins, V. H. da S. (2020). Risk factors and complications of tuberculosis due to treatment abandonment. Research, Society and Development, 9(6), e109963501.

Sousa, G. O., Sales, B. N., Gomes, J. G. F., Silva, M. do A., \& Oliveira, G. A. L. de. (2020). Tuberculosis epidemiology in northeastern Brazil, 2015 - 2019. Research, Society and Development, 9(8), e82985403.

Vachon, J., Gallant, V., \& Siu, W. (2018). Can we eliminate tuberculosis?: Tuberculosis in Canada, 2016. Canada Communicable Disease Report, 44(3-4), 7581 .

Vendramini, S. H. F., Santos, N. S. G. M. D., Santos, M. D. L. S. G., Chiaravalloti Neto, F., Ponce, M. A. Z., Gazetta, C. E., \& Ruffino Netto, A. (2010). Análise espacial da co-infecção tuberculose/HIV: relação com níveis socioeconômicos em município do sudeste do Brasil. Revista da Sociedade Brasileira de Medicina Tropical, 43, 536-541.

World Health Organization. (2017). Global turberculosis report 2017.

Zhu, Y., Wu, J., Feng, X., Chen, H., Lu, H., Chen, L., \& Rui, C. (2017). Patient characteristics and perceived health status of individuals with HIV and tuberculosis coinfection in Guangxi, China. Medicine, 96(14), e6475. 